\title{
Metabolic syndromes and diabetes \\ GW23-e1181 AS TIME GOES BY, ARE ANTHROPOMETRIC MEASURES STILL PREDICTORS OF DIABETES INCIDENCE?
}

doi:10.1136/heartjnl-2012-302920g.1

Chen Xiaoping, Liu Kai, He Sen, Chen Xiaoping. Department of Cardiovascular Medicine, West China Hospital, Sichuan University, Chengdu 610041, China

Objectives The aims of the present study were to examine whether the anthropometric measures could predict diabetes incidence in a Chinese population during a 15 -years follow-up.

Methods The data were collected in 1992 and then again in 2007 from the same group of 687 employees. Waist circumference, body mass index, waist to hip ratio and waist to height ratio were collected base on a standard protocol. To assess the effects of baseline anthropometric measures on the new onset of diabetes, logistic regression models were used to estimate the ORs of them, and the discriminatory power of anthropometric measures for diabetes was assessed by the area under the receiver operating curve (AROC).

Results Of the 687 individuals without diabetes at baseline, 74 individuals were diagnosed with diabetes during a 15-year followup period (incidence 10.8\%). These anthropometric measures also predicted future diabetes during a long follow-up $(p<0.001)$. The areas under the ROC curves were 0.668 (95\% CI 0.601 to 0.734 ) for BMI, 0.701 (95\% CI 0.641 to 0.760 ) for WC, 0.693 (95\% CI 0.637 to 0.748 ) for WHpR and 0.716 (95\% CI 0.657 to 0.774 ) for WHtR, respectively (all $\mathrm{p}<0.001$ ).

Conclusions These anthropometric measures could predict diabetes with different duration of follow-up time. They should be recommended in most clinical practices. 